\title{
Intravenous lipid rescue and ropivacaine systemic toxicity
}

\author{
Fateh Bazerbachi $\cdot$ Kevin Rank $\cdot$ Aaron Chan
}

Received: 12 April 2013/Accepted: 21 June 2013/Published online: 10 July 2013

(C) Japanese Society of Anesthesiologists 2013

Keywords Ropivacaine - Intralipids · Resuscitation · Local anaesthetics toxicity

To the Editor:

Serious cardiac toxicity following ropivacaine infusion is rare. Side effects that responded to lipid infusions have been described [1].

A 57-year-old man with impaired liver function and end-stage renal disease (ESRD) was admitted for an belowknee amputation. Postoperatively, ropivacaine catheters were placed.

Later, the patient became lethargic, and bradycardic, with tinnitus, dysgeusia, and hallucinations. EKG showed widened QRS and prolonged QT without ST changes (Supplementary Figure 1a, b). Echocardiography revealed an ejection fraction (EF) of $20 \%$ without wall motion abnormality. Peak troponin-I-ES $=4.3$. We estimated the infusion to be $1,540 \mathrm{mg}$ ropivacaine.

A total of $2,480 \mathrm{ml}$ intralipids $20 \%$, and one round of plasmapheresis, were given with transient response. The patient ultimately died of cardiac arrest (Supplementary Table 1.)

Cardiac toxicity has been reversed using infusion of lipids in animal models. Reports have suggested its clinical efficacy in humans [1]. The mechanism of lipid rescue is

Electronic supplementary material The online version of this article (doi:10.1007/s00540-013-1669-6) contains supplementary material, which is available to authorized users.

F. Bazerbachi $(\bowtie) \cdot$ K. Rank · A. Chan

Department of Medicine, University of Minnesota,

420 Delaware Street SE, MMC 284, Minneapolis,

MN 55455, USA

e-mail: fateh.b@gmail.com; bazer001@umn.edu thought to be a combination of reduced tissue binding by reestablished equilibrium in a plasma lipid phase and a beneficial energetic-metabolic effect. Ropivacaine may be less responsive to lipid rescue therapy because of its decreased lipophilicity. Slow accumulation into tissues may be more difficult to reverse than a sudden bolus [2].

We have evidence of ropivacaine toxicity considering the Naranjo algorithm score of 6 , which correlates to the category of a probable adverse drug reaction. The pharmacokinetics are not affected by renal failure, and the liver accounts for the majority of clearance [3]. In this patient, the impaired liver was further hypoperfused, considering myocardial depression. This report suggests the failure of lipid rescue.

Conflict of interest None.

\section{References}

1. Litz RJ, Popp M, Stehr SN, Koch T. Successful resuscitation of a patient with ropivacaine-induced asystole after axillary plexus block using lipid infusion. Anaesthesia. 2006;61(8):800-1.

2. Kuo I, Akpa BS. Validity of the lipid sink as a mechanism for the reversal of local anesthetic systemic toxicity: a physiologically based pharmacokinetic model study. Anesthesiology. 2013;118: 1350-61.

3. Pere PJ, Ekstrand A, Salonen M, Honkanen E, Sjövall J, Henriksson J, Rosenberg PH. Pharmacokinetics of ropivacaine in patients with chronic renal failure. Br J Anesth. 2011;106(4): 512-21. 